\title{
Crystal structure of pentachloro(3,3'-thiodipropionitrile)tantalum(V), $\mathrm{TaCl}_{5}\left[\mathrm{NC}\left(\mathrm{CH}_{2}\right)_{2} \mathrm{~S}\left(\mathrm{CH}_{2}\right)_{2} \mathrm{CN}\right]$ and of pentachloro(3,3'-thiodipropio- nitrile)niobium( $(\mathrm{V}), \mathrm{NbCl}_{5}\left[\mathrm{NC}\left(\mathrm{CH}_{2}\right)_{2} \mathrm{~S}\left(\mathrm{CH}_{2}\right)_{2} \mathrm{CN}\right]$
}

Ibrahim-Abdelhalim-Ahmed, R. Blachnik, H. Reuter and K. Stumpf

Universität Osnabrück, Institut für Chemie, Barbarastr. 7, D-49069 Osnabrück, Germany

Received August 12, 1998, CSD-No. 409362 and CSD-No. 409363

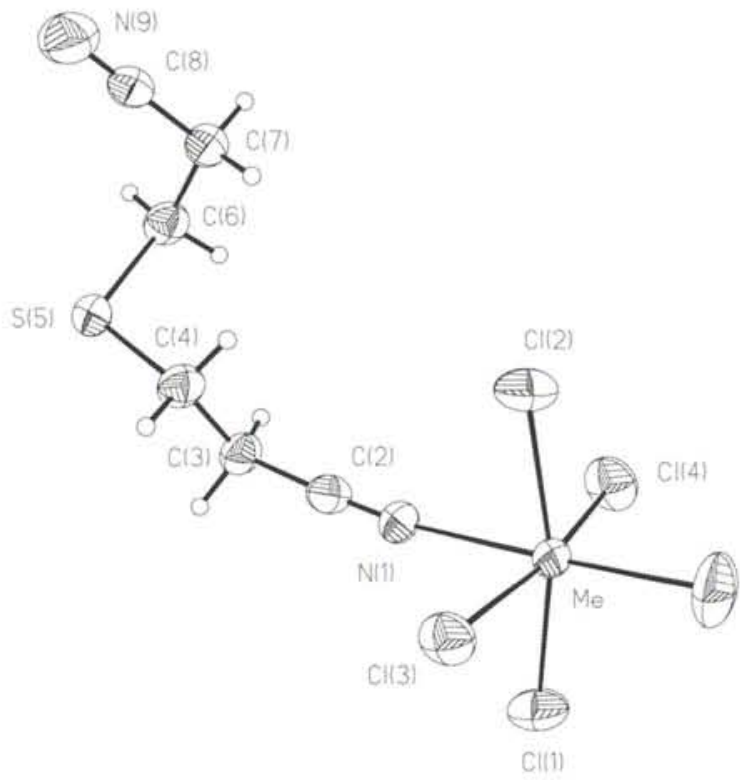

Abstract

$\mathrm{C}_{6} \mathrm{H}_{8} \mathrm{Cl}_{5} \mathrm{~N}_{2} \mathrm{ST}$ a, monoclinic, $P 12_{1} / n 1$ (No. 14), $a=8.051$ (3) $\AA$, $b=9.430(2) \AA, c=18.293(5) \AA, \beta=100.32(3)^{\circ}, V=1366.4 \AA^{3}$, $Z=4, R_{\mathrm{gt}}(F)=0.031, R_{\mathrm{w}}\left(F^{2}\right)=0.073, T=223 \mathrm{~K}$.

$\mathrm{C}_{6} \mathrm{H}_{8} \mathrm{Cl}_{5} \mathrm{~N}_{2} \mathrm{NbS}$, monoclinic, $P 121 / n 1$ (No. 14), $a=8.069$ (1) $\AA$, $b=9.4689(9) \AA, c=18.440(2) \AA, \beta=100.19(1)^{\circ}, V=1386.7 \AA^{3}$, $Z=4, R_{\mathrm{gt}}(F)=0.020, R_{\mathrm{w}}\left(F^{2}\right)=0.057, T=293 \mathrm{~K}$.

\section{Source of material}

The title compounds were prepared using standard Schlenk techniques. Niobium(V) chloride or tantalum(V) chloride and 3,3'-thiodipropionitrile were dissolved in dichloromethane. The solution was covered with a layer of $n$-hexane. Solvent diffusion occured at room temperature over a period of days and resulted in yellow and red crystals for niobium(V) chloride and tanta$\operatorname{lum}(\mathrm{V})$ chloride, respectively. Both compounds are sensitive to moisture.

\author{
Discussion \\ Structure data reveal that both compounds are isotypic with \\ almost the same bond lengths and bond angles. The ligand mo- \\ lecule coordinates via only one nitrogen atom $(\bar{d}(\mathrm{Nb}-\mathrm{Cl})=$ \\ $2.249 \AA, d(\mathrm{Nb}-\mathrm{N})=2.259 \AA ; \bar{d}(\mathrm{Ta}-\mathrm{Cl})=2.232 \AA, d(\mathrm{Ta}-\mathrm{N})=$ \\ $2.239 \AA$ ). The neutral complex is a distorted octahedra centred \\ with the metal atom. Due to the high moisture sensitivity other \\ investigation methods such as D.S.C. could not be applied. The \\ first complexes between niobium(V) and tantalum(V) halides \\ with dinitriles have been reported in [1]. \\ 1. Pentachloro( $3,3^{\prime}$-thiodipropionitrile)tantalum(V), \\ $\mathrm{TaCl}_{5}\left[\mathrm{NC}\left(\mathrm{CH}_{2}\right)_{2} \mathrm{~S}\left(\mathrm{CH}_{2}\right)_{2} \mathrm{CN}\right]$ \\ Table 1. Data collection and handling.

\begin{tabular}{|c|c|}
\hline $\begin{array}{l}\text { Crystal: } \\
\text { Wavelength: }\end{array}$ & $\begin{array}{l}\text { yellow needle, size } 0.31 \times 0.46 \times 0.86 \mathrm{~mm} \\
\text { Mo } K_{\alpha} \text { radiation }(0.71073 \AA)\end{array}$ \\
\hline$\mu:$ & $91.44 \mathrm{~cm}^{-1}$ \\
\hline Diffractometer, scan mode: & 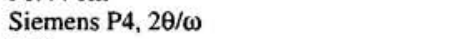 \\
\hline $2 \theta_{\max }:$ & $50^{\circ}$ \\
\hline$N(h k l)_{\text {measured, }} N(h k l)_{\text {unique: }}$ & 3325,2411 \\
\hline Criterion for $I_{\mathrm{obs}}, N(\mathrm{hkl})_{\mathrm{gt}}$ : & $I_{\text {obs }}>2 \sigma\left(I_{\text {obs }}\right), 1914$ \\
\hline$N(\text { param })_{\text {refined: }}$ & 138 \\
\hline Programs: & SHELXS-86 [2], SHELXL-93 [3] \\
\hline
\end{tabular}

Table 2. Atomic coordinates and displacement parameters (in $\AA^{2}$ ).

\begin{tabular}{llllll}
\hline Atom & Site & $x$ & $y$ & $z$ & $U_{\text {iso }}$ \\
\hline $\mathrm{H}(31)$ & $4 e$ & $0.6586(9)$ & $0.3128(7)$ & $0.1745(4)$ & $0.040(9)$ \\
$\mathrm{H}(32)$ & $4 e$ & $0.7872(9)$ & $0.4072(7)$ & $0.2284(4)$ & $0.040(9)$ \\
$\mathrm{H}(41)$ & $4 e$ & $1.003(1)$ & $0.3305(8)$ & $0.1573(4)$ & $0.040(9)$ \\
$\mathrm{H}(42)$ & $4 e$ & $0.872(1)$ & $0.2178(8)$ & $0.1195(4)$ & $0.040(9)$ \\
$\mathrm{H}(61)$ & $4 e$ & $1.044(1)$ & $0.3467(7)$ & $0.3134(4)$ & $0.040(9)$ \\
$\mathrm{H}(62)$ & $4 e$ & $1.136(1)$ & $0.2132(7)$ & $0.3523(4)$ & $0.040(9)$ \\
$\mathrm{H}(71)$ & $4 e$ & $1.245(1)$ & $0.3668(7)$ & $0.2370(4)$ & $0.040(9)$ \\
$\mathrm{H}(72)$ & $4 e$ & $1.334(1)$ & $0.3707(7)$ & $0.3205(4)$ & $0.040(9)$
\end{tabular}


Table 3. Atomic coordinates and displacement parameters (in $\AA^{2}$ ).

\begin{tabular}{lllllllllll}
\hline Atom & Site & $x$ & $y$ & $z$ & $U_{11}$ & $U_{22}$ & $U_{33}$ & $U_{12}$ & $U_{13}$ & $U_{23}$ \\
\hline $\mathrm{Ta}(1)$ & $4 e$ & $0.73978(3)$ & $0.75545(3)$ & $0.00680(2)$ & $0.0174(2)$ & $0.0185(2)$ & $0.0185(2)$ & $-0.0004(1)$ & $-0.0005(1)$ & $0.0013(1)$ \\
$\mathrm{Cl}(1)$ & $4 e$ & $0.4494(2)$ & $0.7692(2)$ & $-0.0012(1)$ & $0.0193(9)$ & $0.046(1)$ & $0.032(1)$ & $0.0061(8)$ & $0.0003(8)$ & $-0.0026(9)$ \\
$\mathrm{Cl}(2)$ & $4 e$ & $1.0243(2)$ & $0.6998(2)$ & $0.0298(1)$ & $0.0168(9)$ & $0.050(1)$ & $0.041(1)$ & $0.0006(8)$ & $0.0010(8)$ & $0.000(1)$ \\
$\mathrm{Cl}(3)$ & $4 e$ & $0.6943(2)$ & $0.5838(2)$ & $-0.0864(1)$ & $0.033(1)$ & $0.0318(9)$ & $0.030(1)$ & $0.0003(8)$ & $0.0019(8)$ & $-0.0089(8)$ \\
$\mathrm{Cl}(4)$ & $4 e$ & $0.7741(3)$ & $0.8908(2)$ & $0.1142(1)$ & $0.042(1)$ & $0.037(1)$ & $0.031(1)$ & $-0.0055(9)$ & $0.0008(9)$ & $-0.0119(8)$ \\
$\mathrm{Cl}(5)$ & $4 e$ & $0.7651(3)$ & $0.9380(2)$ & $-0.0702(1)$ & $0.068(2)$ & $0.032(1)$ & $0.044(1)$ & $0.002(1)$ & $0.015(1)$ & $0.0160(9)$ \\
$\mathrm{N}(1)$ & $4 e$ & $0.7260(8)$ & $0.5732(6)$ & $0.0839(4)$ & $0.025(3)$ & $0.021(3)$ & $0.038(4)$ & $0.001(3)$ & $0.003(3)$ & $0.005(3)$ \\
$\mathrm{C}(2)$ & $4 e$ & $0.7370(9)$ & $0.4834(7)$ & $0.1240(4)$ & $0.019(4)$ & $0.018(3)$ & $0.037(4)$ & $0.000(3)$ & $0.005(3)$ & $0.002(3)$ \\
$\mathrm{C}(3)$ & $4 e$ & $0.7615(9)$ & $0.3681(7)$ & $0.1786(4)$ & $0.025(4)$ & $0.025(4)$ & $0.024(4)$ & $0.000(3)$ & $-0.001(3)$ & $0.009(3)$ \\
$\mathrm{C}(4)$ & $4 e$ & $0.908(1)$ & $0.2716(8)$ & $0.1647(4)$ & $0.027(4)$ & $0.030(4)$ & $0.019(4)$ & $0.001(3)$ & $-0.002(3)$ & $0.001(3)$ \\
$\mathrm{S}(5)$ & $4 e$ & $0.9776(2)$ & $0.1505(2)$ & $0.2399(1)$ & $0.029(1)$ & $0.0228(9)$ & $0.038(1)$ & $-0.0013(8)$ & $-0.0017(9)$ & $0.0047(8)$ \\
$\mathrm{C}(6)$ & $4 e$ & $1.108(1)$ & $0.2631(7)$ & $0.3053(4)$ & $0.027(4)$ & $0.026(4)$ & $0.025(4)$ & $0.003(3)$ & $0.000(3)$ & $-0.001(3)$ \\
$\mathrm{C}(7)$ & $4 e$ & $1.273(1)$ & $0.3104(7)$ & $0.2819(4)$ & $0.030(4)$ & $0.018(3)$ & $0.038(4)$ & $-0.002(3)$ & $-0.001(4)$ & $-0.007(3)$ \\
$\mathrm{C}(8)$ & $4 e$ & $1.382(1)$ & $0.1940(9)$ & $0.2684(4)$ & $0.027(4)$ & $0.033(4)$ & $0.033(4)$ & $-0.003(4)$ & $-0.001(3)$ & $-0.007(4)$ \\
$\mathrm{N}(9)$ & $4 e$ & $1.4657(9)$ & $0.1038(8)$ & $0.2580(5)$ & $0.034(4)$ & $0.043(5)$ & $0.074(6)$ & $0.008(4)$ & $0.000(4)$ & $-0.021(4)$ \\
& & & & & & & & & & \\
\end{tabular}

\section{Pentachloro( $3,3^{\prime}$-thiodipropionitrile)niobium(V), $\mathrm{NbCl}_{5}\left(\mathrm{NC}\left(\mathrm{CH}_{2}\right)_{2} \mathrm{~S}\left(\mathrm{CH}_{2}\right)_{2} \mathrm{CN}\right]$}

Table 4. Data collection and handling.

\begin{tabular}{|c|c|}
\hline Crystal: & light red needle, size $0.06 \times 0.03 \times 0.23 \mathrm{~mm}$ \\
\hline Wavelength: & Mo $K_{\alpha}$ radiation $(0.71073 \AA)$ \\
\hline$\mu:$ & $19.51 \mathrm{~cm}^{-1}$ \\
\hline Diffractometer, scan mode: & 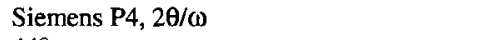 \\
\hline $2 \theta_{\max }$ & $44^{\circ}$ \\
\hline$N(h k l)_{\text {measured, }} N(h k l)_{\text {unique: }}$ & 2330,1608 \\
\hline Criterion for $I_{\mathrm{obs}}, N(h k l)_{\mathrm{gt}}$ : & $I_{\mathrm{obs}}>2 \sigma\left(I_{\mathrm{obs}}\right), 1524$ \\
\hline$N(\text { param })_{\text {refined: }}$ & 138 \\
\hline Programs: & SHELXS-86 [2], SHELXL-93 [3] \\
\hline
\end{tabular}

Table 5. Atomic coordinates and displacement parameters (in $\AA^{2}$ ).

\begin{tabular}{llllll}
\hline Atom & Site & $x$ & $y$ & $z$ & $U_{\text {iso }}$ \\
\hline H(3A) & $4 e$ & $0.7899(4)$ & $1.0927(3)$ & $0.2280(2)$ & $0.048(3)$ \\
H(3B) & $4 e$ & $0.6604(4)$ & $1.1857(3)$ & $0.1748(2)$ & $0.048(3)$ \\
H(4A) & $4 e$ & $0.8686(4)$ & $1.2790(3)$ & $0.1193(2)$ & $0.048(3)$ \\
H(4B) & $4 e$ & $1.0001(4)$ & $1.1676(3)$ & $0.1566(2)$ & $0.048(3)$ \\
H(6A) & $4 e$ & $1.1361(4)$ & $1.2872(3)$ & $0.3505(2)$ & $0.048(3)$ \\
H(6B) & $4 e$ & $1.0446(4)$ & $1.1536(3)$ & $0.3129(2)$ & $0.048(3)$ \\
H(7A) & $4 e$ & $1.3324(4)$ & $1.1303(3)$ & $0.3203(2)$ & $0.048(3)$ \\
H(7B) & $4 e$ & $1.2439(4)$ & $1.1315(3)$ & $0.2375(2)$ & $0.048(3)$ \\
& & & & & \\
\hline
\end{tabular}

Table 6. Atomic coordinates and displacement parameters (in $\AA^{2}$ ).

\begin{tabular}{|c|c|c|c|c|c|c|c|c|c|c|}
\hline Atom & Site & $x$ & $y$ & $z$ & $U_{11}$ & $U_{22}$ & $U_{33}$ & $U_{12}$ & $U_{13}$ & $U_{23}$ \\
\hline $\mathrm{Nb}(1)$ & $4 e$ & $0.73968(3)$ & $0.74242(2)$ & $0.00664(2)$ & $0.0300(2)$ & $0.0274(2)$ & $0.0274(2)$ & $0.0000(1)$ & $0.0004(1)$ & $-0.0020(1)$ \\
\hline $\mathrm{Cl}(\mathrm{l})$ & $4 e$ & $0.4506(1)$ & $0.73148(9)$ & $-0.00042(5)$ & $0.0324(5)$ & $0.0658(6)$ & $0.0505(6)$ & $-0.0092(4)$ & $0.0002(4)$ & $0.0071(4)$ \\
\hline $\mathrm{Cl}(2)$ & $4 e$ & $1.0233(1)$ & $0.7988(1)$ & $0.03008(5)$ & $0.0295(4)$ & $0.0684(6)$ & $0.0557(6)$ & $-0.0008(4)$ & $0.0015(4)$ & $-0.0002(5)$ \\
\hline $\mathrm{Cl}(3)$ & $4 e$ & $0.6956(1)$ & $0.91499(8)$ & $-0.08519(4)$ & $0.0472(5)$ & $0.0452(5)$ & $0.0423(5)$ & $-0.0001(4)$ & $0.0037(4)$ & $0.0126(4)$ \\
\hline $\mathrm{Cl}(4)$ & $4 e$ & $0.7727(1)$ & $0.60865(9)$ & $0.11349(5)$ & $0.0635(6)$ & $0.0512(5)$ & $0.0434(5)$ & $0.0079(4)$ & $0.0040(4)$ & $0.0166(4)$ \\
\hline $\mathrm{Cl}(5)$ & $4 e$ & $0.7652(1)$ & $0.56155(9)$ & $-0.06966(5)$ & $0.0966(8)$ & $0.0449(5)$ & $0.0582(6)$ & $0.0012(5)$ & $0.0182(5)$ & $-0.0216(4)$ \\
\hline$N(1)$ & $4 e$ & $0.7253(3)$ & $0.9254(3)$ & $0.0839(1)$ & $0.037(2)$ & $0.033(1)$ & $0.033(1)$ & $0.001(1)$ & $-0.000(1)$ & $-0.008(1)$ \\
\hline $\mathrm{C}(2)$ & $4 e$ & $0.7372(4)$ & $1.0145(3)$ & $0.1249(2)$ & $0.030(2)$ & $0.040(2)$ & $0.038(2)$ & $0.002(1)$ & $0.001(1)$ & $0.001(2)$ \\
\hline$C(3)$ & $4 e$ & $0.7632(4)$ & $1.1309(3)$ & $0.1785(2)$ & $0.036(2)$ & $0.037(2)$ & $0.037(2)$ & $-0.001(1)$ & $0.004(1)$ & $-0.013(1)$ \\
\hline$C(4)$ & $4 e$ & $0.9060(4)$ & $1.2260(3)$ & $0.1642(2)$ & $0.042(2)$ & $0.043(2)$ & $0.033(2)$ & $-0.003(1)$ & $-0.002(2)$ & $-0.002(1)$ \\
\hline$S(5)$ & $4 e$ & $0.9770(1)$ & $1.34832(8)$ & $0.23837(5)$ & $0.0390(5)$ & $0.0307(4)$ & $0.0498(5)$ & $0.0004(3)$ & $-0.0037(4)$ & $-0.0060(3)$ \\
\hline$C(6)$ & $4 e$ & $1.1087(4)$ & $1.2366(3)$ & $0.3043(2)$ & $0.043(2)$ & $0.043(2)$ & $0.031(2)$ & $-0.004(1)$ & $-0.001(2)$ & $-0.002(1)$ \\
\hline$C(7)$ & $4 e$ & $1.2709(4)$ & $1.1891(3)$ & $0.2815(2)$ & $0.040(2)$ & $0.036(2)$ & $0.045(2)$ & $-0.000(2)$ & $-0.002(2)$ & $0.004(2)$ \\
\hline $\mathrm{C}(8)$ & $4 e$ & $1.3785(4)$ & $1.3039(4)$ & $0.2671(2)$ & $0.036(2)$ & $0.043(2)$ & $0.046(2)$ & $0.004(2)$ & $-0.006(2)$ & $0.006(2)$ \\
\hline $\mathrm{N}(9)$ & $4 e$ & $1.4634(4)$ & $1.3932(4)$ & $0.2561(2)$ & $0.047(2)$ & $0.064(2)$ & $0.095(3)$ & $-0.008(2)$ & $0.000(2)$ & $0.022(2)$ \\
\hline
\end{tabular}

\section{References}

1. Gill, M. S.; Ahuja, H. S.; Rao, G. S.: Complexes of Niobium(V) and Tantalum(V) Halides with Dinitriles, 1, Malonitrile und Succinonitrile. Inorg. Chim. Act. 7 (1973) 359- 364.
2. Sheldrick, G. M.: SHELXS-86. Program for crystal structure determination. University of Göttingen, Germany 1986.

3. Sheldrick, G. M.: SHELXL-93. Program for crystal structure refinement. University of Göttingen, Germany 1993. 